\title{
Spatiotemporal Neural Activity Changes in the Molluscan Olfactory Center Specifically Induced by Innately Aversive and In Vitro Aversively Conditioned Odors
}

\author{
Kohei Ishida1, Momo Murata', Koharu Hashiguchi'2, Yoshimasa Komatsuzaki², \\ Satoshi Watanabe ${ }^{3}$, Minoru Saito ${ }^{*}$
}

${ }^{1}$ Department of Correlative Study in Physics and Chemistry, Graduate School of Integrated Basic Sciences, Nihon University, Tokyo, Japan

${ }^{2}$ Department of Physics, College of Science and Technology, Nihon University, Tokyo, Japan

${ }^{3}$ Department of Ultrastructural Research, National Institute of Neuroscience, National Center of Neurology and Psychiatry, Tokyo, Japan

Email: ^msaito@chs.nihon-u.ac.jp

How to cite this paper: Ishida, K., Murata, M., Hashiguchi, K., Komatsuzaki, Y., Watanabe, S. and Saito, M. (2018) Spatiotemporal Neural Activity Changes in the Molluscan Olfactory Center Specifically Induced by Innately Aversive and In Vitro Aversively Conditioned Odors. Journal of Behavioral and Brain Science, 8, 294-305. https://doi.org/10.4236/jbbs.2018.85018

Received: March 29, 2018

Accepted: May 14, 2018

Published: May 17, 2018

Copyright $\odot 2018$ by authors and Scientific Research Publishing Inc. This work is licensed under the Creative Commons Attribution International License (CC BY 4.0).

http://creativecommons.org/licenses/by/4.0/

\begin{abstract}
In the procerebrum (PC), the olfactory center, of the land slug Limax, an oscillation of local field potential (LFP) with $0.5-1 \mathrm{~Hz}$ is observed by electrophysiological extracellular recording. The oscillation has a phase delay along the distal-proximal axis, resulting in the propagation of waves from the distal to proximal region. One important advantage of nervous systems of mollusks such as Limax is that their nervous systems in vitro retain several types of computational properties found in vivo (e.g. learning and memory). A previous study showed that the LFP frequency in the PC of Limax increased specifically in response to innately aversive and in vitro aversively conditioned odors. In the present study, we examined spatiotemporal neural activity changes induced in the PC by those odors using the fluorescent voltage imaging technique. The results showed that innately aversive (onion and hexanol) and in vitro aversively conditioned (carrot, which is innately attractive) odors specifically induced an increase in propagation speed of the neural activity in the PC, while innately attractive odors did not induce it. The results also suggested that the avoidance behavior by those odors might be induced by the increase of propagation speed and the following increases in the discharges of the partial nerve that transmits the motor output.
\end{abstract}




\section{Keywords}

Land Slug, Olfactory Center, In Vitro Odor-Aversion Conditioning, Spatiotemporal Neural Activity, Fluorescent Voltage Imaging

\section{Introduction}

The procerebrum (PC) of the land slug Limax is the olfactory center involved in olfactory discrimination, learning and memory [1] [2]. In the PC, an oscillation of local field potential (LFP) with $0.5-1 \mathrm{~Hz}$ is observed by electrophysiological extracellular recording [3]. The oscillatory activity is supposed to arise from synaptic interactions of two types of interneurons [4] [5] [6]. Additionally, previous studies using optical recordings revealed spatiotemporal neural activities in the PC [4] [7] [8] [9] [10]. The oscillation has a phase delay along the distal-proximal axis, while the neural activities along the anterior-posterior axis are synchronized, resulting in the propagation of waves from the distal to proximal region. Some of the previous studies also reported that the oscillations in the PC are modulated in response to odor application to the nose.

One important advantage of nervous systems of mollusks such as Limax is that their nervous systems in vitro retain several types of computational properties found in vivo (e.g. learning and memory). Several types of in vitro conditioning have been reported: classical conditioning of the gill-withdrawal reflex system in Aplysia [11]; classical conditioning in feeding systems in Limax [12], Lymnaea [13] and Aplysia [14] [15]; and operant conditioning in feeding systems in Aplysia [16].

For the olfactory system, one of the present authors reported an in vitro odor-aversion conditioning in the land slug Limax [17] that has the abilities of odor learning in vivo [1]. In the previous study [17], the whole of the central nervous system (CNS) was isolated and an odor-aversion conditioning paradigm was applied to the in vitro preparation. The results showed that the frequency of LFP oscillation in the PC increased specifically in response to aversively conditioned odors that elicited avoidance behavior, which was similar to innately aversive odors. From the LFP, however, only local neural activities around the electrode can be known. Because global neural network properties must be more informative on the olfactory discrimination, learning and memory, we here examined spatiotemporal neural activity changes induced in the PC by those odors using the fluorescent voltage imaging technique.

\section{Methods}

\subsection{Behavioral Experiments}

\section{Odor responses and in vitro odor-aversion conditioning}

We first examined the odor response behaviors although they have been already reported in some previous studies. We used the laboratory-bred land slug 
Limax valentianus. In the physiological experiments mentioned below, we used carrot, cucumber, onion and hexanol as odors. We confirmed whether these odors are attractive or aversive as follows. The slug was put on the center of a glass plate. After acclimation to the surroundings, the slug began to crawl. Then, at about $4 \mathrm{~cm}$ from its head to the crawling direction, the odor source (carrot, cucumber or onion juice, or $0.1 \%$ hexanol) was dropped on a straight line (about $4 \mathrm{~cm}$ in length), and we observed its behavior (Figure 1). When the odor was attractive, the slug approached the odor source. In in vivo odor-aversion conditioning, quinidine sulfate (unconditioned stimulus; UCS) was dropped on its head just before the slug touched the attractive odor source (conditioned stimulus; CS).

\subsection{Physiological Experiments}

\subsubsection{Preparation}

The land slug was anesthetized by injection of $\mathrm{Mg}^{2+}$ solution (composition in mM: $\mathrm{MgCl}_{2} \cdot 6 \mathrm{H}_{2} \mathrm{O} 57.6$, D-glucose 5, HEPES-NaOH 5) into the body cavity, and the central nervous system (CNS) was isolated with the tentacles, the medial lip

(a)
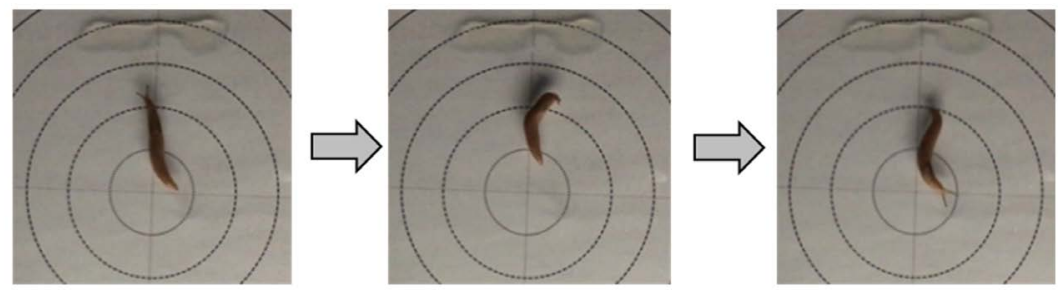

(b)
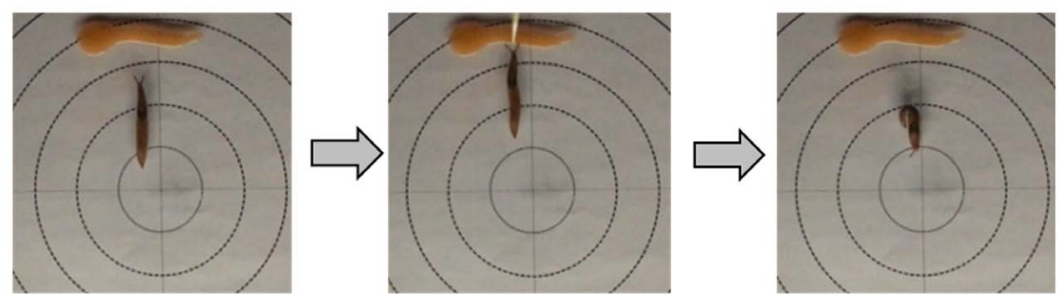

(c)
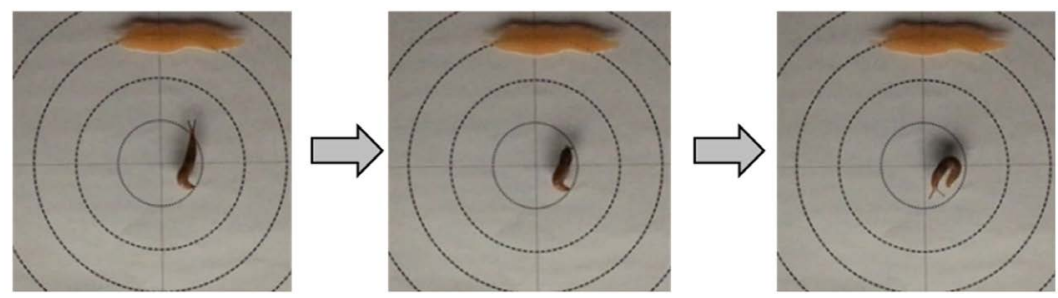

Figure 1. Response behaviors of the slug to (a) the onion odor, (b) the carrot odor and (c) the aversively conditioned carrot odor. In (b), the slug approached to the carrot juice (the left photograph), and then quinidine sulfate was dropped on the head of the slug as unconditioned stimulus (UCS) just before it touched the carrot juice (conditioned stimulus (CS)) (the middle photograph). The supplemental movies can be seen in the web site (http://saitolab-chaos.com/Papers/jbbs2018_1/Supplemental-movie1.html; double speed reproduction). 
nerve, which transmits the gustatory input to the CNS, and the parietal nerve, which transmits the motor output from the CNS, from the body in the dissection solution (composition in $\mathrm{mM}: \mathrm{NaCl} 35, \mathrm{KCl} 2, \mathrm{MgCl}_{2} \cdot 6 \mathrm{H}_{2} \mathrm{O} 28, \mathrm{CaCl}_{2}$ 4.9, D-glucose 5, HEPES-NaOH 5 ( $\mathrm{pH} 7.6)$ ). The PC is located at the distal part of each cerebral ganglion (Figure 2).

\subsubsection{Odor Stimulation and In Vitro Odor-Aversion Conditioning}

Figure 3 shows the schematic illustration of the present experimental apparatus. The isolated CNS was moved to the recording chamber consisted of two compartments with a slit between them. The CNS was placed into one of the compartments filled with the slug saline and one of the superior tentacles was placed through the slit in the other compartment exposed to the air. We paid attention for the nose (olfactory epithelium at the tip of the tentacle) not to be dried during the physiological measurement.

The odors were delivered to the nose with air using our odor stimulator. For in vitro odor-aversion conditioning, we used the attractive odor as the CS. One second after the onset of the odor delivery, the medial lip nerve was electrically stimulated using a glass suction electrode for the UCS. Here, a single pulse ( $3 \mathrm{~V}$, $1 \mathrm{~ms}$ ) was applied with an electronic stimulator (SEN-7203, NIHON KOHDEN, Japan) through an isolator (SS-202J, NIHON KOHDEN, Japan).

\subsubsection{Measurements}

For the fluorescent voltage imaging, a voltage sensitive dye Di-4-ANEPPS (WAKO, Japan) was used. The isolated CNS was loaded for $50 \mathrm{~min}$ at room temperature with $86 \mu \mathrm{M}$ Di-4-ANEPPS in the presence of $0.06 \%$ cremophor EL and $0.6 \%$ ethanol in the slug saline (composition in $\mathrm{mM}: \mathrm{NaCl} 70, \mathrm{KCl} 2$, $\mathrm{MgCl}_{2} \cdot 6 \mathrm{H}_{2} \mathrm{O}$ 4.7, $\mathrm{CaCl}_{2} 4.9$, D-glucose 5, HEPES-NaOH 5 (pH 7.6)). After the dye loading, the preparation was washed with the slug saline.

The stained preparation placed on the recording chamber was mounted on the stage of a microscope (E-FN1, Nikon, Japan) with a $16 \times$ objective lens

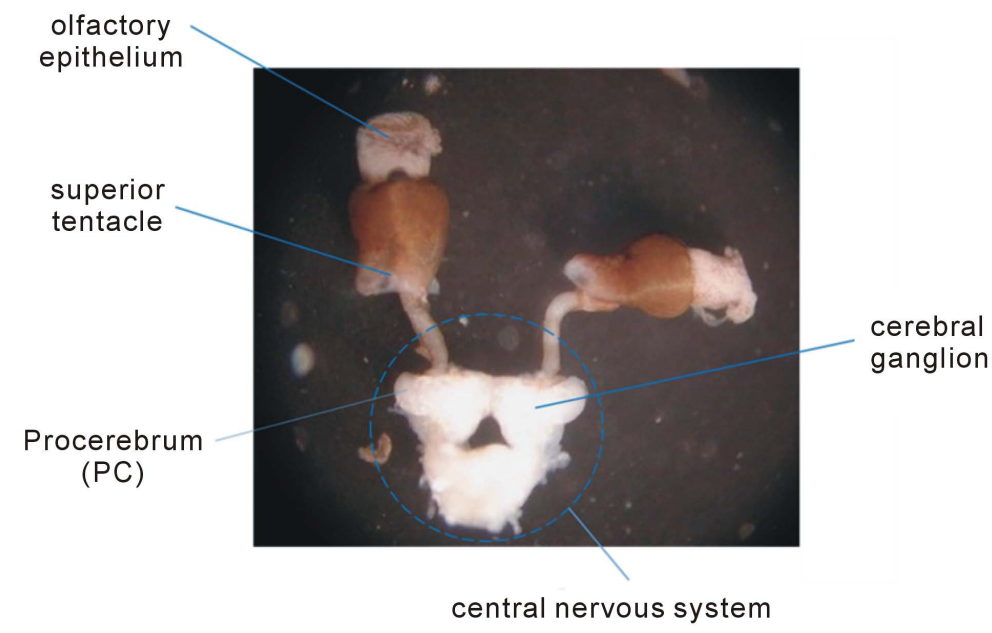

Figure 2. Preparation of the central nervous system of the land slug Limax valentianus. 


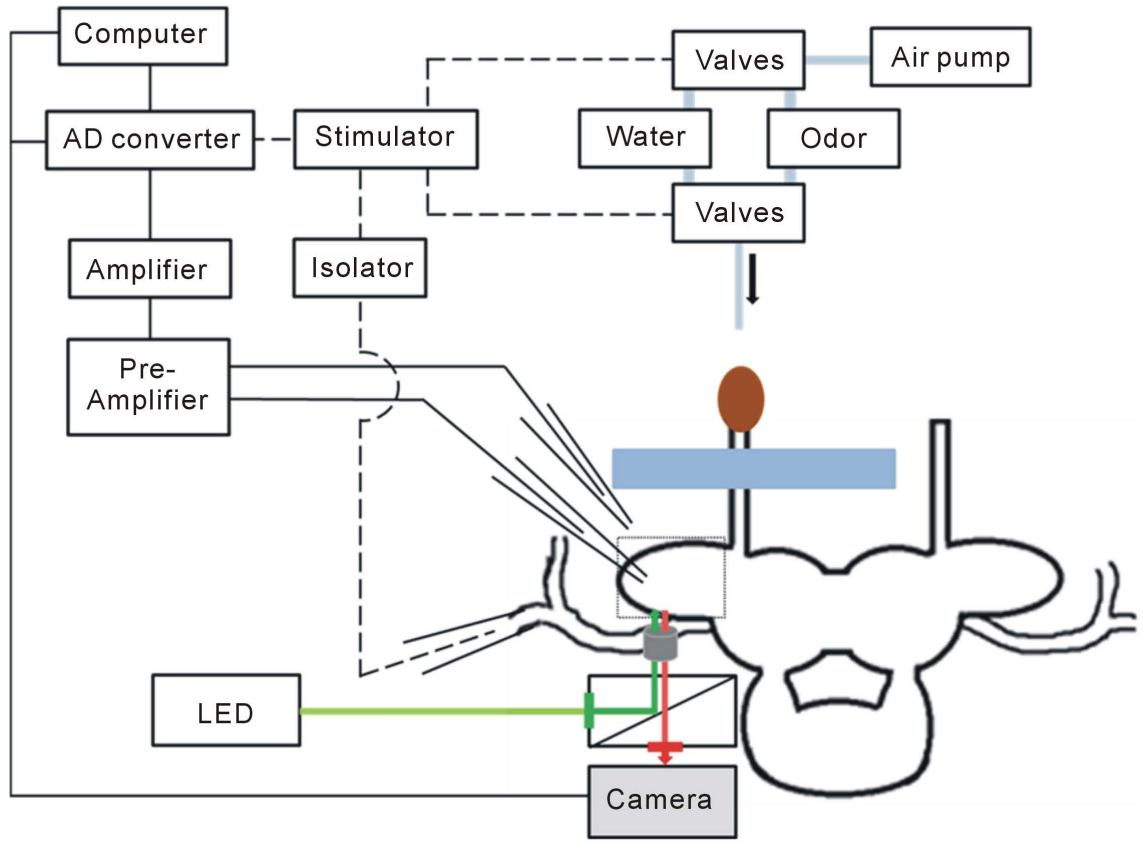

Figure 3. Schematic illustration of the present experimental apparatus.

(water-immersion, $0.8 \mathrm{NA}$, Nikon, Japan). The dye was excited by a LED of 530 $\mathrm{nm}$ with a half width of $25 \mathrm{~nm}$ (LEX2-G, Brain Vision, Japan) through an excitation filter (EX510-560). The emitted fluorescence of $705 \mathrm{~nm}$ was detected through a dichroic mirror (DM575) and a barrier filter (BA590). The fluorescence images were acquired at a rate of $10 \mathrm{~ms} /$ flame by a sCMOS-CCD camera (Zyla, Andor, Ireland). The image sequences were stored into a personal computer (Dell Precision T5600, Dell, USA).

Together with the fluorescent voltage imaging, we simultaneously measured the LFP using a glass suction electrode filled with the slug saline. Alternatively, we measured the activity of the parietal nerve in the same way. The reference electrode was placed into the saline-filled compartment in which the CNS was placed. The signals were amplified with an extracellular recording amplifier (ER-1, Cygnus, USA) and transferred through an AD converter (PowerLab, ADInstruments, Australia) into the personal computer (Dell Precision T5600, Dell, USA) with $1 \mathrm{kHz}$ sampling frequency.

The measurements of odor responses mentioned above were started following about 30 min recovery after the staining, and a series of measurements were performed within 3 hours including the in vitro odor-aversion conditioning procedure. Until a series of measurements were completed, the isolated CNS maintained their function.

\subsection{Statistical Analysis}

Difference between the groups was examined for statistical significance using a paired two tailed Student's $t$-test. $p<0.05$ was assumed to be a significance difference. The data were expressed as mean \pm SEM. 


\section{Results and Discussion}

\subsection{Behavioral Experiments}

Figure 1 shows the response behaviors of the slug to the odors. The slug mostly approached to the carrot and cucumber odor sources and these odors were therefore regarded as innately attractive odors, while it mostly avoided the onion and hexanol odor sources and these odors were therefore regarded as innately aversive odors. Additionally, the slug mostly became to avoid the CS odor source after the in vivo odor-aversion conditioning.

\subsection{Physiological Experiments}

Figure 4 shows the changes in LFP oscillation induced by the hexanol (innately aversive) and carrot (innately attractive) odors, and the in vitro aversively conditioned carrot odor. The hexanol odor much increased the LFP frequency (Figure 4(a)), while the carrot odor did not much change it (Figure 4(b)). For the other

(a)

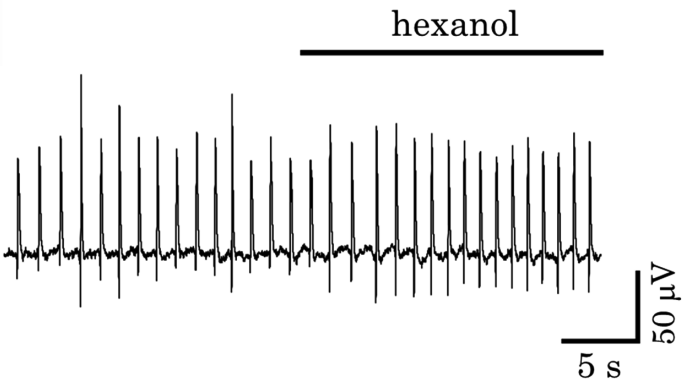

(b)

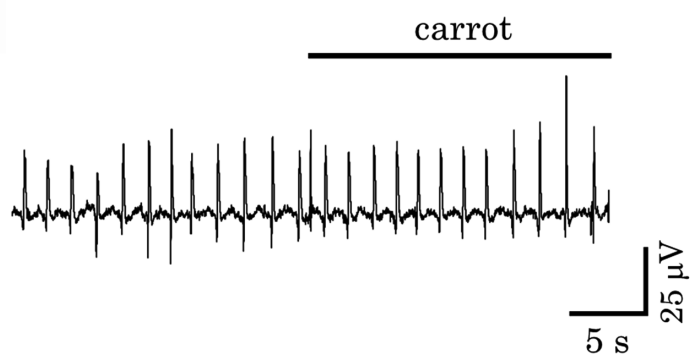

(c)

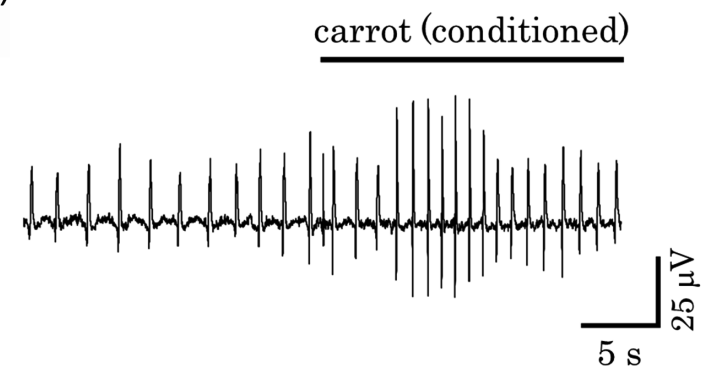

Figure 4. Changes in LFP oscillation induced by (a) the hexanol (innately aversive) odor, (b) the carrot (innately attractive) odor and (c) the in vitro aversively conditioned carrot odor. The measurement for the conditioned odor was preformed about one hour after the conditioning. The horizontal thick black line in each figure shows the period of the odor delivery. 
aversive (onion) and attractive (cucumber) odors, similar results were obtained. The change in LFP frequency induced by each odor is summarized in Figure 5. Here, the LFP frequencies before and after an odor stimulation were estimated as the average frequencies obtained from time intervals of several peaks within $10 \mathrm{~s}$ just before and after the stimulation. Before the odor stimulation, the LFP frequency was $0.5-1 \mathrm{~Hz}$. Compared with it, the increases in LFP frequency were $22.3 \% \pm 2.5 \%(\mathrm{n}=27, p<0.01)$ for the hexanol odor (gray bar in Figure 5$)$ and $7.3 \% \pm 3.1 \%(\mathrm{n}=7)$ for the onion odor (gray bar in Figure 5), while they were $-1.5 \pm 1.4 \%(\mathrm{n}=21)$ for the carrot odor (white bar in Figure 5 ) and $-1.6 \% \pm$ $1.6 \%(\mathrm{n}=7)$ for the cucumber odor (white bar in Figure 5).

Additionally, after the in vitro odor-aversion conditioning was performed for the carrot odor, the CS odor induced an increase in the LFP frequency $(9.1 \% \pm$ 2.3\%) (gray bar in Figure 5; see also Figure 4(c)), which was significantly larger $(p<0.01)$ than that before the conditioning, similarly to the innately aversive odors, but the non-conditioned odor (cucumber) did not induce it $(0.4 \% \pm$ 1.1\%) (gray bar in Figure 5). Here, such a measurement was performed one hour after the conditioning. These results are not inconsistent with the previous result [17], although the UCS was much weaker than that of the previous study (a single pulse of $3 \mathrm{~V}$ in the present study, 100 pulses of $5 \mathrm{~V}$ with $6.67 \mathrm{~Hz}$ in the previous study). Therefore, the same UCS condition was used in the other present experiments mentioned below.

Figure 6 shows a propagation of neural activity (fluorescence change of the voltage sensitive dye) in the PC measured by the fluorescent voltage imaging.

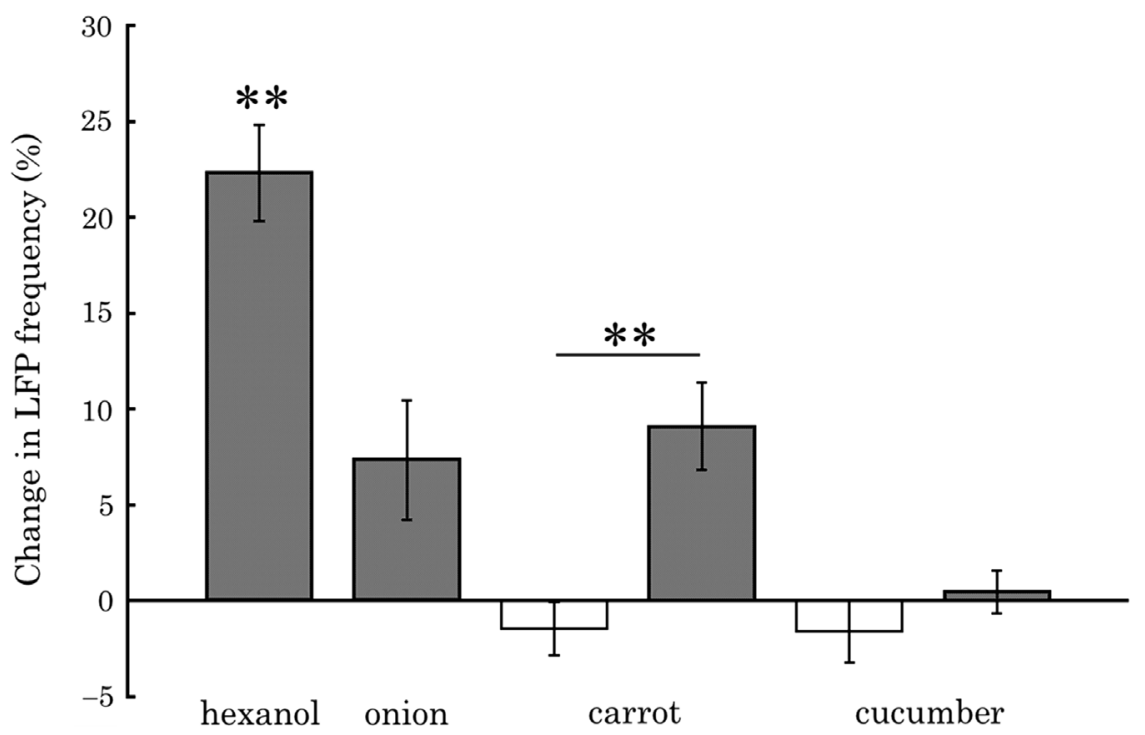

Figure 5. Change in the LFP frequency induced by each odor stimulation. The vertical axis shows the ratio of the LFP frequency to that before the odor stimulation. For carrot and cucumber, the white bars show the data before the in vitro carrot-aversion conditioning and the gray bars show the data after the conditioning. The gray bars should be compared with the data for innately aversive odors, hexanol and onion (gray bars). ${ }^{* *} p<$ 0.01 . 

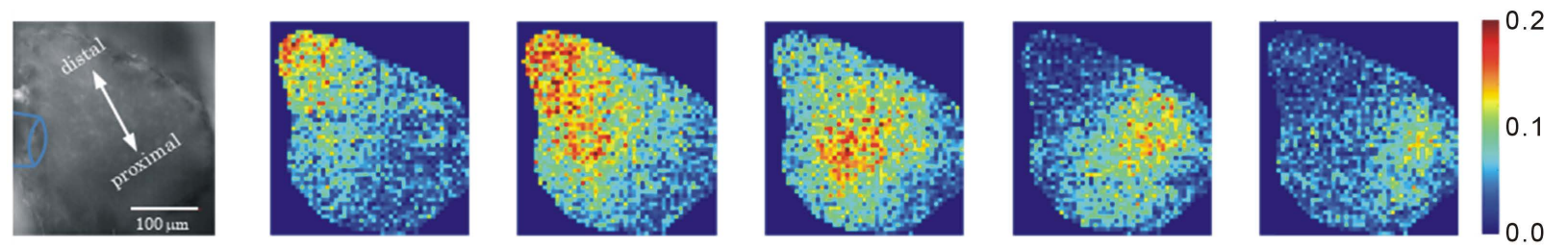

\section{$\frac{0}{\frac{1}{4}}$}

Figure 6. Propagation of neural activity in the PC. Here, no odor stimulation is applied. The left image shows the fluorescence image of the PC. The blue shape is the electrode for the LFP measurement. The sequential pseudo-color images show the fluorescence change of the voltage sensitive dye. The time interval is $0.1 \mathrm{~s}$. The size of one pixel of each image is about $6.5 \mu \mathrm{m} \times 6.5 \mu \mathrm{m}$. The supplemental movie can be seen in the web site (http://saitolab-chaos.com/Papers/jbbs2018_1/Supplemental-movie2.html; $1 / 4$ speed reproduction).

The neural activity propagated from the distal to proximal region in the PC. Here, no odor stimulation is applied. To estimate the propagation speed, the time delay between the peaks of fluorescence change in the distal and proximal regions was used (Figure 7). The change in propagation speed in the PC induced by each odor stimulation is summarized in Figure 8. Here, the propagation speeds before and after an odor stimulation were estimated as the average speeds obtained from several propagations just before and after the stimulation. Before the odor stimulation, the propagation speed was $1-2 \mathrm{~mm} / \mathrm{s}$. Compared with it, the propagation speed much increased by the innately aversive odors; $74.6 \% \pm$ $12.7 \%(\mathrm{n}=14, p<0.01)$ for the hexanol odor (gray bar in Figure 8) and $23.0 \pm$ $14.8 \%(\mathrm{n}=6)$ for the onion odor (gray bar in Figure 8), but not for the innately attractive odors; $4.3 \% \pm 1.5 \%(\mathrm{n}=6)$ for the carrot odor (white bar in Figure 8) and $-8.3 \% \pm 5.3 \%(n=4)$ for the cucumber odor (white bar in Figure 8 ). This specific property for the innately aversive odors was also observed for the in vitro aversively conditioned odors (Figure 8). Figure 9 shows the changes in neural activity propagation before and after the conditioning was performed for the carrot odor. The CS odor induced an increase in propagation speed $(20.8 \% \pm 6.1 \%)$ (gray bar in Figure 8), which was significantly larger $(p<0.05)$ than that before the conditioning, similarly to the innately aversive odors, but the non-conditioned odor (cucumber) did not induce it $(4.7 \% \pm 3.9 \%)$ (gray bar in Figure 8 ). Here, such a measurement was performed one hour after the conditioning.

The previous study showed that the LFP frequency is correlated with the discharges of the parietal nerve and the discharges can be an in vitro index of odor-avoidance behavior [17]. Because the LFP is measured through the electrode put on a position in the PC, it is considered that the increase in LFP frequency is due to the increase in speed of the neural activity propagation repetitively generated in the PC. Additionally, the neural activity must repetitively propagate to the partial nerve that transmits the motor output and the increase in the propagation speed must increase its discharges. In fact, it was observed in the present study (Figure 10) although the statistical analysis has not been performed yet. Thus, the present results suggest that the avoidance behavior by innately aversive and in vivo aversively conditioned odors might be induced by the increase in propagation speed of the neural activity in the PC and the following increases in the discharges of the partial nerve that transmits the motor output. 
(a)

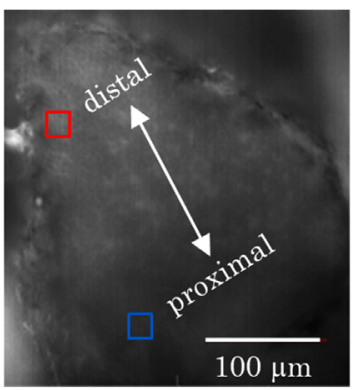

(b)

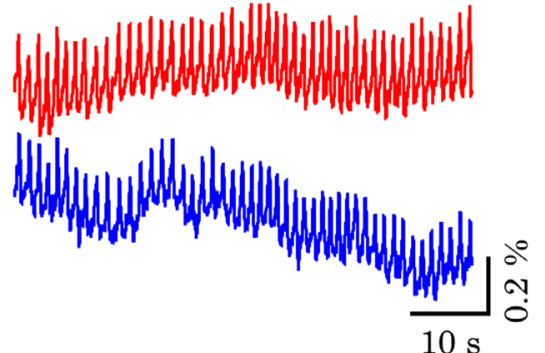

(c)

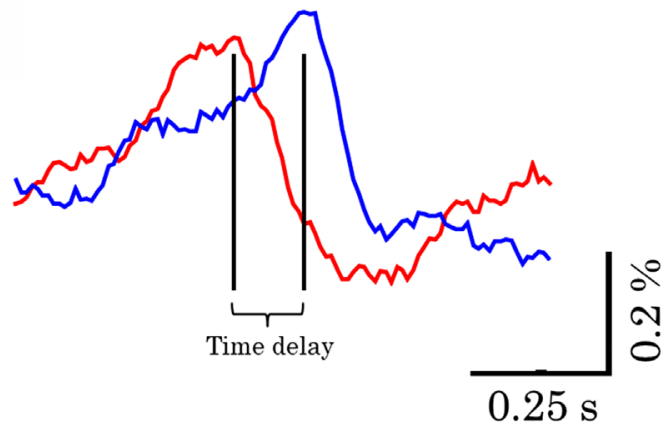

Figure 7. (a) Fluorescence image of the PC. The fluorescence changes were analyzed in the red and blue squares. (b) Time courses of the fluorescence changes in the distal (red) and proximal (blue) regions. (c) Time delay between the peaks of the fluorescence changes in the distal (red) and proximal (blue) regions. The propagation speed of neural activity in the PC was estimated from the time delay.

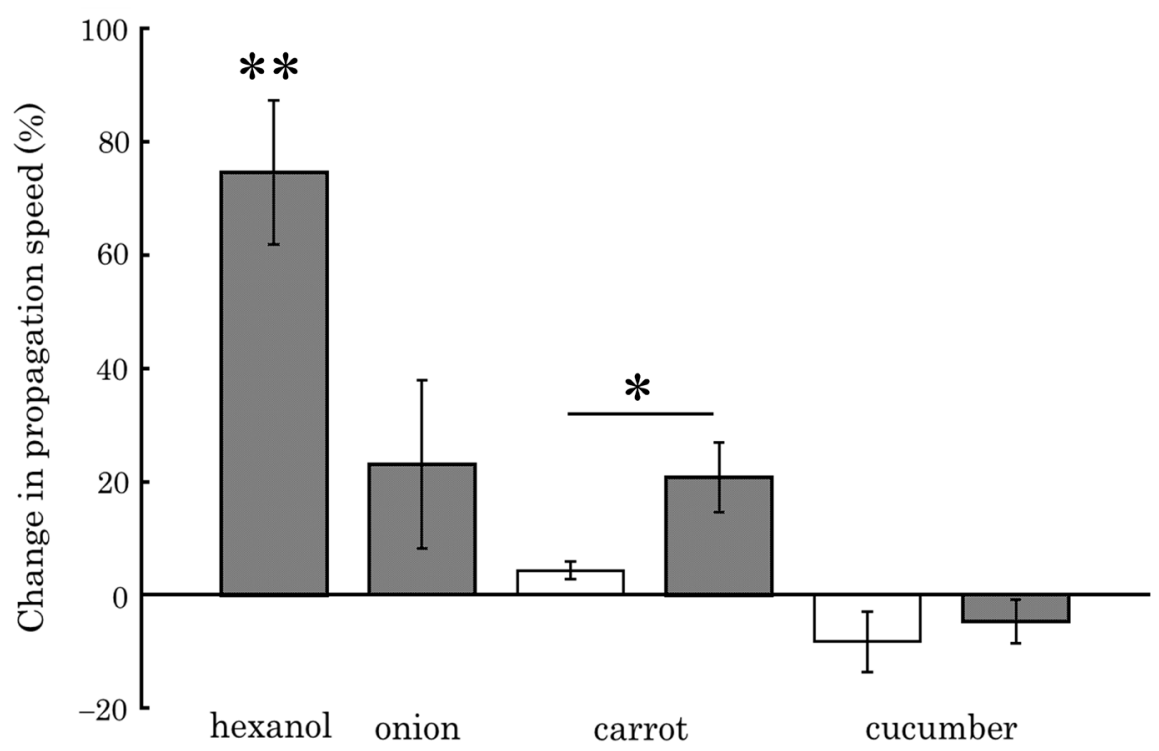

Figure 8. Change in propagation speed of neural activity in the PC induced by each odor stimulation. The vertical axis shows the ratio of the propagation speed to that before the odor stimulation. For carrot and cucumber, the white bars show the data before the in vitro carrot-aversion conditioning and the gray bars show the data after the conditioning. The gray bars should be compared with the data for innately aversive odors, hexanol and onion (gray bars). ${ }^{* *} p<0.01,{ }^{*} p<0.05$. 
(a)
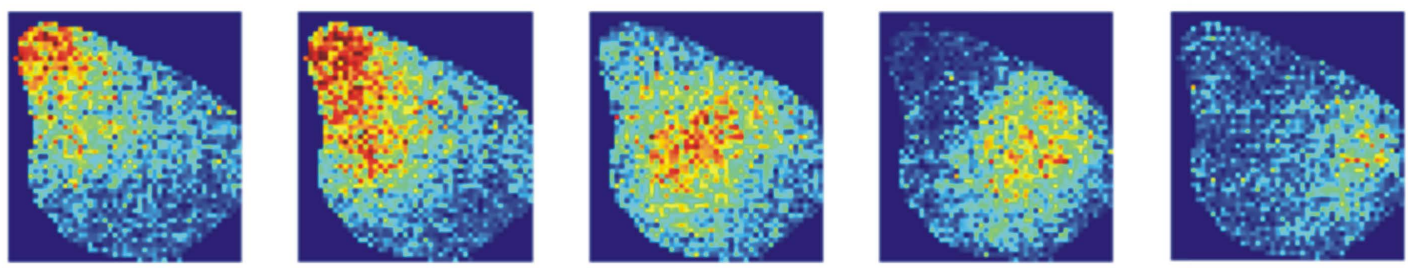

0.2

0.1

$\frac{\frac{2}{2}}{\frac{4}{4}}$

0.0

(b)
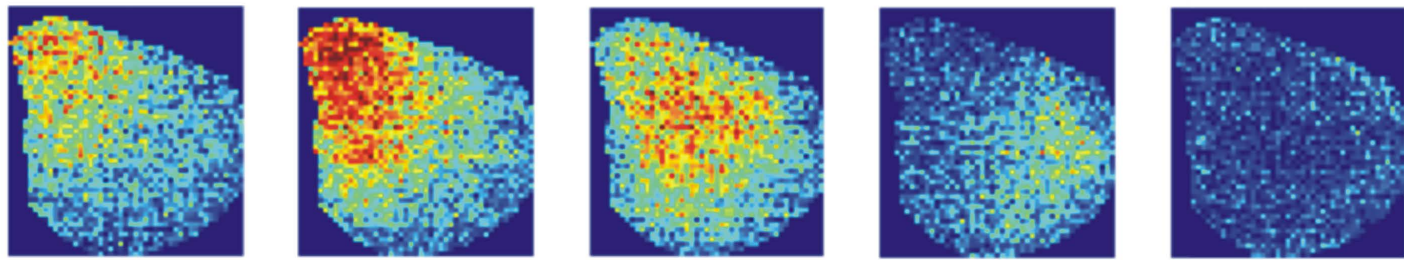

0.2

0.1

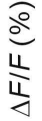

0.0

Figure 9. Propagation of neural activity in the PC induced by the carrot odor (a) before and (b) after the in vitro carrot-aversion conditioning. The pseudo-color images show the fluorescence change of the voltage sensitive dye. The sequential images in each column are shown from about $3 \mathrm{~s}$ after the onset of the odor delivery. The time interval is $0.1 \mathrm{~s}$. The size of one pixel of each image is about $6.5 \mu \mathrm{m} \times 6.5 \mu \mathrm{m}$. The supplemental movies can be seen in the web site

(http://saitolab-chaos.com/Papers/jbbs2018_1/Supplemental-movie3.html; 1/4 speed reproduction. The odor stimulation was applied at $0 \mathrm{~s}$ in each movie.).

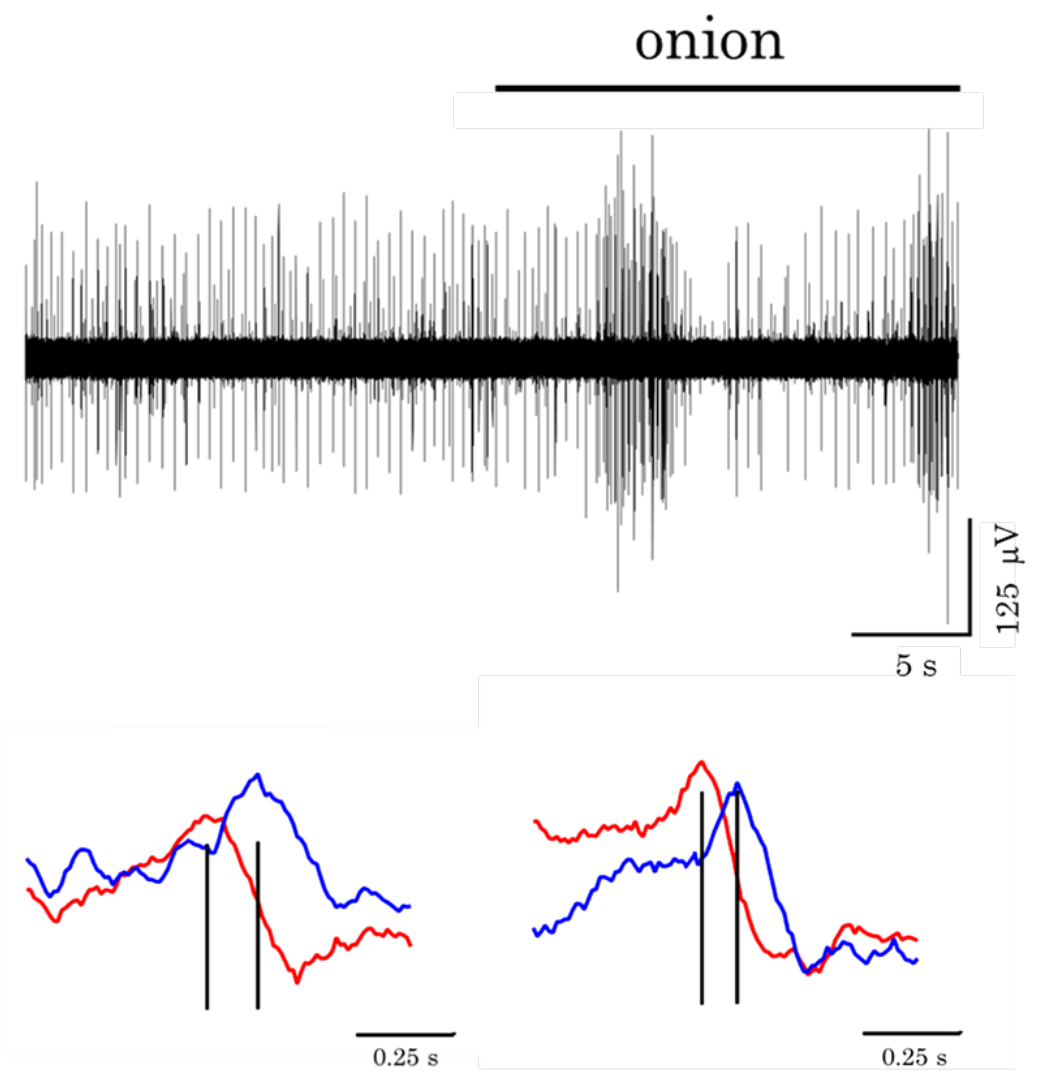

Figure 10. Relation between the discharges of the partial nerve (upper figure) and the fluorescence changes in the distal (red trace) and proximal (blue trace) regions in the PC (lower figure). In the upper figure, the horizontal thick black line shows the period of the onion odor delivery. In the lower figure, the left and right pairs of fluorescence traces were obtained before and after the odor stimulation, respectively. The vertical black lines show the positions of the peaks of the fluorescence change. 


\section{Conclusion}

In the present study, we examined the changes in spatiotemporal neural activity induced in the olfactory center, the procerebrum (PC), of the land slug Limax valentianus by some odors using the fluorescent voltage imaging technique. As a result, innately aversive odors induced the increase in propagation speed of the neural activity in the PC and in vitro aversively conditioned odors also induced the similar phenomenon, while innately attractive odors did not induce it. Therefore, this phenomenon was specific for innately aversive and in vitro aversively conditioned odors. The present results also suggested that the avoidance behavior by those odors might be induced by the increase of propagation speed of the neural activity in the PC and the following increases in the discharges of the partial nerve that transmits the motor output.

\section{References}

[1] Kimura, T., Suzuki, H., Kono, E. and Sekiguchi, T. (1998) Mapping of Interneurons That Contribute to Food Aversive Conditioning in the Slug Brain. Learning \& Memory, 4, 376-388. https://doi.org/10.1101/lm.4.5.376

[2] Teyke, T. and Gelperin, A. (1999) Olfactory Oscillation Augment Odor Discrimination Not Odor Identification by Limax CNS. NeuroReport, 10, 1061-1068. https://doi.org/10.1097/00001756-199904060-00030

[3] Gelperin A. and Tank, D.W. (1990) Odour-Modulated Collective Network Oscillations of Olfactory Interneurons in a Terrestrial Mollusk. Nature, 345, 437-440. https://doi.org/10.1038/345437a0

[4] Kleinfeld, D., Delaney, K.R., Fee, M.S., Flores, J.A., Tank, D.W. and Gelperin, A. (1994) Dynamics of Propagating Waves in the Olfactory Network of a Terrestrial Mollusk: An Electrical and Optical Study. Journal of Neurophysiology, 72, 1402-1419. https://doi.org/10.1152/jn.1994.72.3.1402

[5] Watanabe, S., Kawahara, S. and Kirino, Y. (1998) Morphological Characterization of the Bursting and Nonbursting Neurons in the Olfactory Centre of the Terrestrial Slug Limax marginatus. Journal of Experimental Biology, 201, 925-930.

[6] Watanabe, S. and Kirino, Y. (2007) Selective Calcium Imaging of Olfactory Interneurons in a Land Mollusk. Neuroscience Letters, 417, 246-249.

https://doi.org/10.1016/j.neulet.2007.02.083

[7] Delaney, K.R., Gelperin, A., Fee, M.S., Flores, A., Gervais, R., Tank, D.W. and Kleinfeld, D. (1994) Waves and Stimulus-Modulated Dynamics in an Oscillating Olfactory Network. Proceedings of National Academy of Sciences of USA, 91, 669-673. https://doi.org/10.1073/pnas.91.2.669

[8] Inoue, S., Kawahara, S., Toda, S., Watanabe, S. and Kirino, Y. (1998) Selective Optical Recording of the Neural Activity in the Olfactory Center of the Land Slug Using a Calcium Indicator Dye. Bioimages, 6, 59-67.

[9] Hamasaki, Y., Hosoi, M., Nakada, S., Shimokawa T. and Saito, M. (2013) Fluorescent Voltage Imaging Technique for the Measurement of Molluscan Neural Activities. Open Journal of Biophysics, 3, 54-58.

https://doi.org/10.4236/ojbiphy.2013.31A007

[10] Watanabe, S., Takanashi, F., Ishida, K., Kobayashi, S., Kitamura, Y., Hamasaki, Y. and Saito, M. (2015) Nitric Oxide-Mediated Modulation of Central Network Dy- 
namics during Olfactory Perception. PLoS One, 10, e0136846.

https://doi.org/10.1371/journal.pone.0136846

[11] Lukowiak, K. and Sahley, C. (1981) The In Vitro Classical Conditioning of a Gill Withdrawal Reflex in Aplysia: Neural Correlates and Possible Neural Mechanism. Science, 212, 1516-1518. https://doi.org/10.1126/science.212.4502.1516

[12] Chang, J.J. and Gelperin A. (1980) Rapid Taste-Aversive Learning by an Isolated Molluscan Central Nervous System. Proceedings of National Academy of Sciences of USA, 77, 6204-6206. https://doi.org/10.1073/pnas.77.10.6204

[13] Kemenes, G., Staras, K. and Benjamin, P.R. (1997) In Vitro Appetitive Classical Conditioning of the Feeding Response in the Pond Snail Lymnaea stagnalis. Journal of Neurophysiology, 78, 2351-2362. https://doi.org/10.1152/jn.1997.78.5.2351

[14] Mozzachiodi, R., Lechner, H.A., Baxter, D.A. and Byrne, J.H. (2003) In Vitro Ana$\log$ of Classical Conditioning of Feeding Behavior in Aplysia. Learning \& Memory, 10, 478-494. https://doi.org/10.1101/lm.65303

[15] Reyes, F.D., Mozzachiodi, R., Baxter, D.A. and Byrne, J.H. (2005) Reinforcement in an In Vitro Analog of Appetitive Classical Conditioning of Feeding Behavior in Aplysia: Blockade by a Dopamine Antagonist. Learning \& Memory, 12, 216-220. https://doi.org/10.1101/lm.92905

[16] Nargeot, R., Baxter, D.A. and Byrne, J.H. (1997) Contingent-Dependent Enhancement of Rhythmic Motor Patterns: An In Vitro Analog of Operant Conditioning. Journal of Neuroscience, 17, 8093-8105. https://doi.org/10.1523/JNEUROSCI.17-21-08093.1997

[17] Inoue, T., Murakami, M., Watanabe, S., Inokuma, Y. and Kirino, Y. (2006) In Vitro Odor-Aversion Conditioning in a Terrestrial Mollusk. Journal of Neurophysiology, 95, 3898-3903. https://doi.org/10.1152/jn.00853.2005 